\title{
Pattern Formation by Lateral Inhibition with Feedback: a Mathematical Model of Delta-Notch Intercellular Signalling
}

\author{
Joanne R. Collier $\dagger$, Nicholas A. M. Monk $\uparrow \S$, Philip K. Maini $\dagger$ and \\ JuLIAN H. LEWIS:* \\ $\dagger$ Centre for Mathematical Biology, Mathematical Institute, University of Oxford, 24-29 St. \\ Giles', Oxford OX13LB, and the $\$ I C R F$ Developmental Biology Unit, Department of \\ Zoology, University of Oxford, South Parks Road, Oxford OX1 3PS, U.K.
}

(Received on 5 March 1996, Accepted in revised form on 2 August 1996)

\begin{abstract}
In many developing tissues, adjacent cells diverge in character so as to create a fine-grained pattern of cells in contrasting states of differentiation. It has been proposed that such patterns can be generated through lateral inhibition - a type of cell-cell interaction whereby a cell that adopts a particular fate inhibits its immediate neighbours from doing likewise. Lateral inhibition is well documented in flies, worms and vertebrates. In all of these organisms, the transmembrane proteins Notch and Delta (or their homologues) have been identified as mediators of the interaction-Notch as receptor, Delta as its ligand on adjacent cells. However, it is not clear under precisely what conditions the Delta-Notch mechanism of lateral inhibition can generate the observed types of pattern, or indeed whether this mechanism is capable of generating such patterns by itself. Here we construct and analyse a simple and general mathematical model of such contact-mediated lateral inhibition. In accordance with experimental data, the model postulates that receipt of inhibition (i.e. activation of Notch) diminishes the ability to deliver inhibition (i.e. to produce active Delta). This gives rise to a feedback loop that can amplify differences between adjacent cells. We investigate the pattern-forming potential and temporal behaviour of this model both analytically and through numerical simulation. Inhomogeneities are self-amplifying and develop without need of any other machinery, provided the feedback is sufficiently strong. For a wide range of initial and boundary conditions, the model generates fine-grained patterns similar to those observed in living systems.
\end{abstract}

(C) Academic Press Limited

\section{Introduction}

In many developing tissues, both in animals and in plants, a fine-grained mixture of different cell types is generated through cell-cell interactions within a population of cells that are initially equivalent in their developmental potential (Greenwald \& Rubin, 1992). Somehow, a scattered subset of cells in the population become singled out for one fate, while their immediate neighbours are consigned to another. Studies in insects and nematode worms, and more recently in vertebrates, have revealed an evolutionarily con-

$\S$ Author to whom correspondence should be addressed.

E-mail: nmonk@maths.ox.ac.uk

*Current address: Imperial Cancer Research Fund, Vertebrate Development Lab., 44 Lincoln's Inn Fields, London WC2A 3PX. served mechanism by which this may come about in animal tissues (Sternberg, 1993; Artavanis-Tsakonas et al., 1995; Chitnis, 1995; Lewis, 1996). The mechanism is based on lateral inhibition - a cell-cell interaction whereby a cell heading for a particular fate inhibits its neighbours from developing in the same way (Goriely et al., 1991; Heitzler \& Simpson, 1991). In the developing nervous system, for example, it has been found that nascent neural cells inhibit their neighbours from becoming committed to a neural fate. In this way, adjacent cells are forced to develop differently.

Genes that mediate lateral inhibition are identifiable by a striking and characteristic mutant phenotype: when they are defective, lateral inhibition fails, and all the cells in the initial population develop 
in the same way, corresponding to the fate of an isolated cell. This default fate - the neural fate, in the case of developing nervous tissue-is termed the primary fate; that of cells that suffer lateral inhibition is called the secondary fate (Sternberg, 1993). Early studies focused on the neuroectoderm of the Drosophila embryo, where failure of lateral inhibition leads to vast overproduction of neurons at the expense of epidermal cells (Lehmann et al., 1983; Campos-Ortega, 1993). Genes identified as responsible for lateral inhibition in this system were thus termed "neurogenic genes"; but it has become clear that the same genes mediate lateral inhibition in a similar way in a great variety of other tissues, both neural and non-neural, both in invertebrates and in vertebrates. Examples range from the gut epithelium of the fly (Tepass \& Hartenstein, 1995) and the gonad of the nematode worm (Wilkinson et al., 1994) to the retina of the chicken (Austin et al., 1995) and the neural plate of the frog (Chitnis et al., 1995). In some cases, the initial population of equivalent cells within which lateral inhibition operates is small-about six cells for a proneural cluster in the neuroectoderm of Drosophila (Skeath \& Carroll, 1992)-and lateral inhibition singles out just one cell within it for the primary fate; in other cases, such as the Drosophila endoderm (Tepass \& Hartenstein, 1995), the initial population is large and lateral inhibition singles out many cells within it, each surrounded by a halo of inhibition, creating a fine-grained spacing pattern.

In either case, lateral inhibition, as outlined above, explains why the neighbours of a cell that is singled out for the primary fate do not adopt that same fate themselves. But can it also explain how the cell that adopts the primary fate becomes singled out in the first place? Or does one have to postulate some other, additional machinery to initiate cell diversification within the original population of equivalent cells? In this paper we explore the proposition that lateral inhibition by itself is sufficient, provided that it is regulated in an appropriate way. The form of the required regulation is simple: the more intense the inhibition a cell receives, the weaker its ability to deliver inhibition must become. This results in a feedback: the more inhibition a cell delivers to its neighbours, the less inhibition it receives back from them, and the more it is consequently able to deliver. The suggestion is that, as a result, any initial slight difference between the neighbours will become self-amplifying, generating a full-blown spatial pattern of inhomogeneity. This mechanism, which we shall call lateral inhibition with feedback, has been proposed in several experimental contexts (Goriely et al., 1991; Heitzler \& Simpson, 1991; Sternberg,
1993) and is supported by evidence that inhibition received does indeed diminish the ability to deliver inhibition (Wilkinson et al., 1994; Heitzler et al., 1996). But formal proof that the mechanism will do what it is proposed to do is still lacking, and so far as we know there has not been any mathematical analysis of its expected temporal behaviour or spatial pattern-forming capabilities.

Advances in knowledge of the underlying biochemistry allow us to formulate these problems in terms of specific molecules. Many of the molecules that implement lateral inhibition with feedback have been identified as products of specific neurogenic genes. In particular, extensive genetic and biochemical studies have identified the product of the neurogenic gene Notch (and its homologues in organisms other than the fly) as the receptor for lateral inhibition, and the product of the neurogenic gene Delta (and its homologues) as the corresponding ligand, expressed in the cell delivering inhibition (reviewed in Artavanis-Tsakonas et al., 1995; Muskavitch, 1994). Both Notch and Delta are transmembrane proteins. The details of the way in which Delta binding leads to activation of Notch remain unclear, but there is increasing evidence to suggest that binding of Delta in the membrane of one cell to Notch in the membrane of another activates Notch by triggering cleavage of its intracellular domain (Lieber et al., 1993; Jarriault et al., 1995; reviewed in Lewis, 1996). Alternatively, Delta binding could activate Notch in some other way. Whatever the precise nature and result of the Delta-Notch interaction, it seems clear that Notch activation stimulates production of proteins of the Enhancer-of-split (E(spl)) family, which in turn are thought to inhibit both commitment to the primary fate and production of Delta activity (reviewed in Lewis, 1996).

In this paper we develop a simple mathematical model to investigate the pattern-forming potential of a system in which lateral inhibition with feedback is mediated by Delta-Notch signalling between adjacent cells. We make only minimal assumptions about the specific details of the biochemical machinery involved. Analytical results and numerical simulations show that, provided the feedback is sufficiently strong (in a sense that we make mathematically precise), differences between neighbouring cells will indeed be self-amplifying, leading to fine-grained spatial patterns of the expected type: cells with high levels of Delta activity and low levels of Notch activation (corresponding to the primary fate) will arise, scattered amongst cells with low Delta activity levels and high Notch activation levels (corresponding to the secondary fate). A simple parameter change-for 
example, in the lifetime of activated Notch or Delta protein - can switch the system from a condition in which the homogeneous condition, with all cells inhibiting one another, is stable to small perturbations, to one in which it is unstable and will amplify small inhomogeneities leading to a spatial pattern of primary fate and secondary fate cells. Less obviously, a system that satisfies the criterion for instability but starts off approximately homogeneous in its spatial pattern may often move towards a spatially homogeneous unstable equilibrium, in which all cells inhibit one another, before developing a spatially inhomogeneous pattern of primary fate and secondary fate cells.

The model presented here provides a simple and versatile framework in which to investigate the effects, not only of lateral inhibition, but also of other mechanisms that may collaborate with the lateral inhibition machinery to control patterns of differentiation. In Section 2 we present our model and give the assumptions it embodies. Section 3 contains analytical and numerical results for the model, with details of the analysis given in Appendices A and B. In Appendix A, we analyse the two-cell (or period 2) system, which gives important understanding of the behaviour of the model. Appendix B contains an extension of the linear analysis developed by Turing (1952) for systems of discrete cells. It allows us to predict the patterns that emerge on one- and two-dimensional infinite domains from small perturbations about the uniform steady state. In Section 4, we discuss the nature and results of our model and biological evidence surrounding the mechanism of Delta-Notch mediated lateral inhibition. The main conclusions of our modelling are presented in Section 5 .

\section{The Model}

We model Delta-Notch signalling in terms of a system of cells each of which is characterised at any instant by just two parameters: the level of Notch activation, $N$, reflecting the intensity of the inhibition the cell experiences, and the level of Delta activity, $D$, reflecting the intensity of the inhibitory signal that it delivers to its neighbours. For present purposes, the details of the biochemistry do not matter greatly, and there is some freedom in how we interpret $N$ and $D$. $N$, for example, could be taken to stand for the quantity of "activated Notch" protein in the cell, meaning the quantity of the active intracellular Notch fragment, if we suppose Notch activation to occur through proteolytic cleavage; or it could be interpreted to mean the amount of the inhibitory transcription factors of the $\mathrm{E}(\mathrm{spl})$ family that are present as a result of Notch activation, or simply the quantity of Notch-Delta complexes formed by binding of Notch to its activating ligand Delta.

Our model embodies the following assumptions:

(1) Cells interact through Delta-Notch signalling only with cells with which they are in direct contact.

(2) The rate of production of Notch activity is an increasing function of the level of Delta activity in neighbouring cells.

(3) The rate of production of Delta activity is a decreasing function of the level of activated Notch in the same cell.

(4) Production of Notch and Delta activity is balanced by decay, described by simple exponential decay with fixed rate constants.

(5) The level of activated Notch in a cell determines the cell's fate: low levels lead to adoption of the primary fate, high levels to adoption of the secondary fate.

These postulates result in a feedback loop between adjacent cells, illustrated in Fig. 1. Denoting the levels of Notch and Delta activity in cell $P$ by $N_{P}$ and $D_{P}$ respectively, we write

$$
\begin{aligned}
& \frac{\mathrm{d}\left(N_{P} / N_{0}\right)}{\mathrm{d} \tau}=F\left(\bar{D}_{P} / D_{0}\right)-\mu N_{P} / N_{0}, \\
& \frac{\mathrm{d}\left(D_{P} / D_{0}\right)}{\mathrm{d} \tau}=G\left(N_{P} / N_{0}\right)-\rho D_{P} / D_{0},
\end{aligned}
$$

where $\tau=$ time, $F:[0, \infty) \rightarrow[0, \infty)$ is a continuous increasing function, $G:[0, \infty) \rightarrow[0, \infty)$ is a continuous decreasing function. We define $f:=F / \mu$ and $g:=G / \rho$; then $N_{0}$ and $D_{0}$ are typical levels of Notch and Delta activity respectively, chosen such that $f$ and $g$ are of order 1. $\mu$ and $\rho$ are positive constants - the rate constants (inverse lifetimes) for decay of Notch

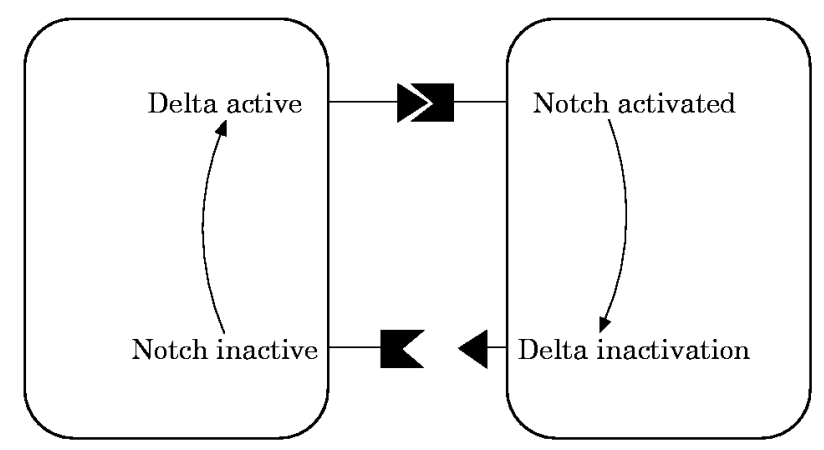

FIG. 1. Diagrammatic representation of the effective feedback loop between Notch and Delta in neighbouring cells. Details of the Notch signalling pathway are omitted for clarity. Key: $\rightarrow$ Delta; $\checkmark$ Notch. 

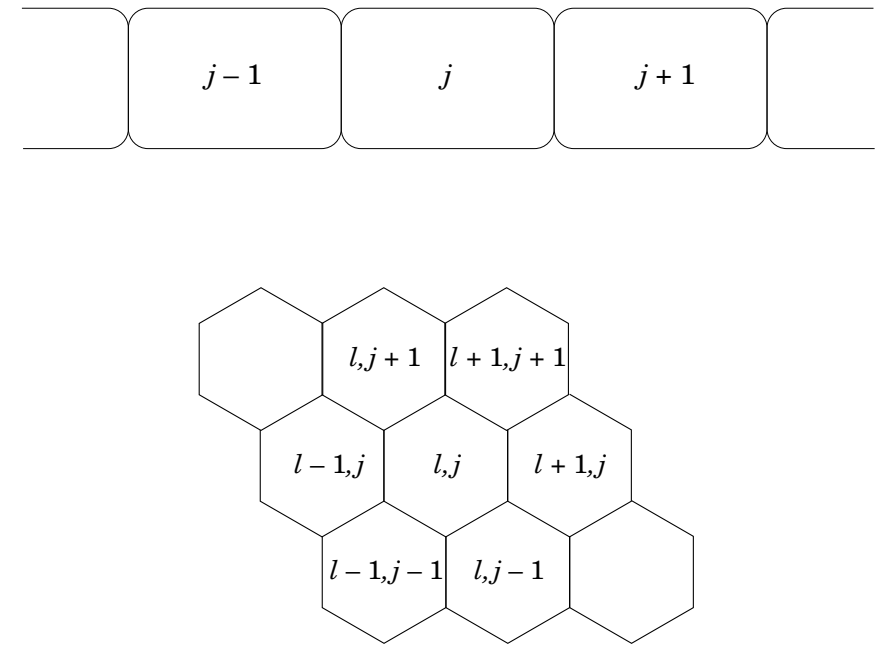

FIG. 2. The labelling scheme used for cells in linear and two-dimensional arrays.

and Delta activity respectively. $\mu$ is thus a measure of the speed of response of levels of Notch activity to changes in Delta activity, while $\rho$ is a measure of the speed of response of levels of Delta activity to changes in Notch activity. $\bar{D}_{P}$ denotes the mean of the levels of Delta activity in the cells adjacent to cell $P$. Thus, for a line of cells indexed by a single number $j$, $\bar{D}_{j}=\left(D_{j-1}+D_{j+1}\right) / 2$. More generally,

$$
\bar{D}_{P}=\frac{1}{r} \sum_{P^{\prime}} D_{P^{\prime}},
$$

where the sum is taken over the immediate neighbours of cell $P$, and $r$ is the total number of such neighbours (see Fig. 2 for specific cell labelling schemes). $f\left(\bar{D}_{P} / D_{0}\right)$ is the equilibrium value of $N_{P} / N_{0}$ when $\bar{D}_{P}$ is held fixed; likewise, $g\left(N_{P} / N_{0}\right)$ is the equilibrium value of $D_{P} / D_{0}$ when $N_{P}$ is held fixed. $\mu f\left(\bar{D}_{P} / D_{0}\right)$ can be interpreted as the rate of production of Notch activity in cell $P$; thus $f\left(\bar{D}_{P} / D_{0}\right)$ can be regarded as the amount of Notch activity produced in a time equal to the mean lifetime of Notch activity. Similarly, $\rho g\left(N_{P} / N_{0}\right)$ can be interpreted as the rate of production of Delta activity in cell $P$, and $g\left(N_{P} / N_{0}\right)$ can be regarded as the amount of Delta activity produced in a time equal to the mean lifetime of Delta activity.

The system (1) can be cast in non-dimensional form by setting $n_{P}=N_{P} / N_{0}, \quad d_{P}=D_{P} / D_{0}, \quad t=\mu \tau$, and $v=\rho / \mu$. Letting 'denote $\mathrm{d} / \mathrm{d} t$, (1) becomes:

$$
\begin{gathered}
\dot{n}_{P}=f\left(\bar{d}_{P}\right)-n_{P}, \\
\dot{d}_{P}=v\left\{g\left(n_{P}\right)-d_{P}\right\},
\end{gathered}
$$

for each cell P. $v$ is the ratio of the decay rates of Delta and Notch activities, and is thus a measure of the relative time-scales over which the levels of Notch and Delta activity vary.

We neglect cell division. This is justified by the fact that in practice, cell cycle times are significantly greater than the time taken for the Delta-Notch mediated fate decision (Hartenstein \& Posakony, 1990).

\section{Results}

ANALYSIS OF THE TWO-CELL SYSTEM

Since the Delta-Notch interaction is local, the analysis of a system consisting of just two cells gives valuable insight into the behaviour of the feedback mechanism in more extensive arrays of cells. Furthermore, the two-cell system with periodic boundary conditions is equivalent to an infinite line of cells in which alternate cells are identical. The details of this analysis are presented in Appendix A. The main conclusions are as follows:

(1) The homogeneous steady state is unstable if the feedback is sufficiently strong. There always exists exactly one homogeneous steady state $\left(n_{1}=n_{2}=n^{*}, d_{1}=d_{2}=d^{*}\right)$, in which the levels of Delta and Notch activity are the same in both cells. The linear stability (i.e. stability to small perturbations) of this state is determined by the strength of the feedback between the cells, which is determined by the functional forms of $f$ and $g$. Specifically, with periodic boundary conditions the homogeneous steady state becomes unstable when

$$
f^{\prime}\left(d^{*}\right) g^{\prime}\left(n^{*}\right)<-1,
$$

where ' denotes differentiation. Similar relations are obtained with fixed boundary conditions. Clearly, a shift in the biochemical parameters underlying the model-for example, a change in the ratio of decay and production rates of Notch or Delta activities - can switch the system from a condition in which the homogeneous steady state is linearly stable to one in which it is not.

(2) When the homogeneous steady state is unstable, the system has a pair of inhomogeneous steady states. When the above instability condition is satisfied, perturbations of the homogeneous steady state are self-amplifying, and the system will diverge from the homogeneous steady state. In fact, whenever the homogeneous steady state is linearly unstable, there exists a pair of heterogeneous steady states, in general stable, such that 
one cell has high Notch activity and low Delta activity, while the other has high Delta activity and low Notch activity.

The behaviour of the system is easiest to see in the limiting cases where $v$ is either very large (so that $d$ adjusts very rapidly compared to $n$ ) or very small (so that $n$ adjusts very rapidly compared to $d$ ). In these cases, the four-dimensional phase space of the full system reduces approximately to two-dimensions. Fig. A2 (Appendix A) illustrates the time evolution of the system in these limits. It is also possible to visualise the dynamics of the system when levels of Notch and Delta activity are roughly equal in the two cells. When this is so, two-dimensional phase portraits can be constructed at each instant, showing the rates of change of the differences in Notch and Delta activities between the cells at that instant. These "instantaneous phase portraits", together with analytical results on the growth rate of the differences in activities between the cells, give a qualitative picture of the behaviour of the two-cell system near homogeneity (see Appendix A and Fig. A3).

\section{NUMERICAL RESULTS}

For systems consisting of more than two cells, we investigate the behaviour of the system numerically. For this purpose, we solve the non-dimensional equations (2) using a Runge-Kutta-Merson method implemented by the NAG routine D02BBF. We take $f$ and $g$ to be of the form:

$$
f(x)=\frac{x^{k}}{a+x^{k}}, g(x)=\frac{1}{1+b x^{h}},
$$

for $x \geqslant 0$, with $a, b>0$ and $k, h \geqslant 1$. The boundary conditions used are either zero Delta activity on the boundary (representing lack of Delta activity in cells outside the domain) or periodic (representing a periodic pattern on an infinite domain). Initial levels of Notch and Delta activity are taken as almost homogeneous and relatively high (i.e. $n_{P}(0)$ and $d_{P}(0)$ are close to one for all cells $P$ ).

The parameter values used to generate the illustrations below are:

$$
a=0.01, b=100, v=1, k=h=2 .
$$

Simulations using these parameters exhibit representative behaviour of the system (2) when heterogeneous steady states exist. With these parameters, the homogeneous steady state of the two-cell (or period 2) system is linearly unstable, and there is a pair of heterogeneous steady states (see above and Appendix A). Simulations using other parameter values yield similar results to those shown here (data not shown).

\section{Line of cells}

We first consider a linear array of $m$ cells with boundary conditions $d_{0}=d_{m+1}=0$. Typical results for the case $m=2$ are shown in Fig. 3. The levels of Notch and Delta activity in the two cells remain similar until they approach the (unstable) homogeneous steady state levels, after which they diverge to the (stable) heterogeneous steady states. When equilibrium has been attained, Cell 1 has a low level of Notch activity and hence adopts the primary fate, while Cell 2 has a high level of Notch activity and adopts the secondary fate. In simulations using parameters for which the system (2) has only one (homogeneous) steady state, the levels of Notch and Delta activity in both cells converge towards this steady state (data not shown).

When there are many cells in a line, the default pattern appears to be one of alternating high and low levels of Notch activity. This pattern often emerges close to the boundaries at first and then spreads inwards, although it sometimes simultaneously develops in the middle of the domain, independently of the boundary conditions. Our numerical simulations reveal that the detailed time evolution to the final alternating pattern depends more strongly on the initial conditions than the boundary conditions. Defects can occur in this alternating pattern, where two adjacent cells have similar high levels of Notch activity, but in all the one-dimensional simulations we have carried out, we have never seen two neighbouring cells with low levels of Notch activity. This finding is in concordance with the experimental result that primary fate cells are always separated by at least one secondary fate cell when lateral inhibition is operative, while secondary fate cells are often found in contact (see, for example, Sternberg, 1993). An example is shown in Fig. 4, with 70 cells.

Simulations such as those shown in Figs 3 and 4 highlight another interesting feature of the system. Suppose the system starts off in a state that is not a steady state, but is spatially more or less homogeneous. This corresponds to the biologically interesting case where the cells are initially equivalent. Suppose, moreover, that the instability criterion (3) is satisfied, so that the homogeneous steady state is unstable. Then for some plausible forms of the functions $f$ and $g$, spatial perturbations will initially decay or grow only slowly compared with other changes in the system. In this way, the system can closely approach the homogeneous steady state before developing significant spatial inhomogeneity. 
A qualitative understanding of this phenomenon, which can be clearly seen in Fig. 4, can be gained from an analysis of the instantaneous rate of change of perturbations about homogeneity. The analysis for the two-cell system is presented in Appendix A.

\section{Two-dimensional array of hexagonal cells}

The qualitative nature of patterns of cell fate on a two-dimensional array can be characterised by the ratio of the number of cells adopting the secondary fate to the number of cells adopting the primary fate. On an infinite domain of hexagonal cells, periodic steady state solutions of (2) exist in which cells adopting the two fates form a regular pattern with a ratio of 2, 3, 4, 5 or 6 . Examples of these patterns are illustrated in Fig. 5.

A pattern like that in Fig. 5(b) can be obtained on a $7 \times 7$ array of hexagonal cells with zero boundary conditions. Similar patterns, but with defects, can emerge on $8 \times 8$ arrays of cells [see Fig. 6(a)].

We have found that the boundary conditions can exert a major influence on the patterns formed in such small domains. Hence we have investigated the
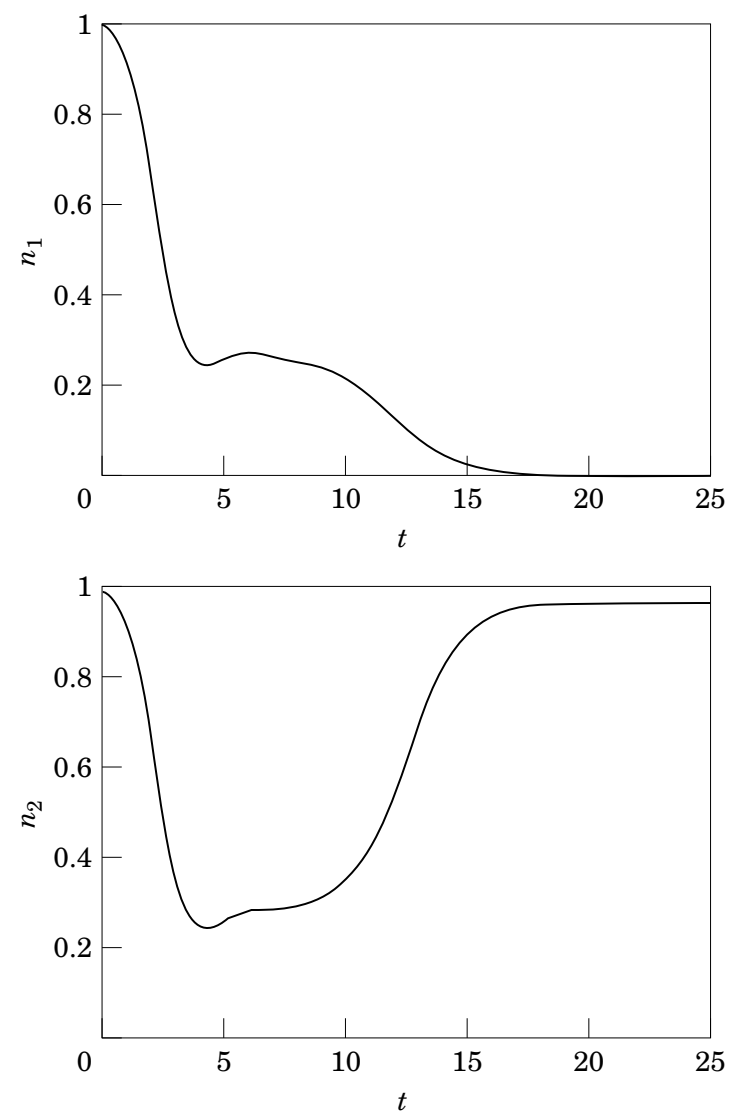

patterns formed on $6 \times 6$ or $7 \times 7$ arrays of hexagonal cells with periodic boundary conditions. This enables us to consider periodic patterns on an infinite domain.

On a $6 \times 6$ array, patterns with periods $1,2,3$ or 6 in $i$ and $j$ are possible. It is the period 3 pattern, illustrated in Fig. 5(a), that emerges when the parameters are given by (5) and the levels of Notch and Delta activity are all close to one initially. A $7 \times 7$ array with periodic boundary conditions should favour the period 7 pattern in Fig. 5(c), but with the parameters in (5) and fairly homogeneous initial conditions, the default pattern still appears to be 5(a), with a secondary to primary fate ratio of two [see Fig. 6(b)]. Pattern 5(c) can, however, be achieved by using initial conditions that are biased towards it.

\section{EFFECT OF BOUNDARY CONDITIONS AND PARAMETERS}

For small domains, boundary conditions and the number of cells in the domain can have a major effect on the emergent pattern of cell fates. In general, the steady-state levels become apparent first near the boundary, and then spread inwards. However, for
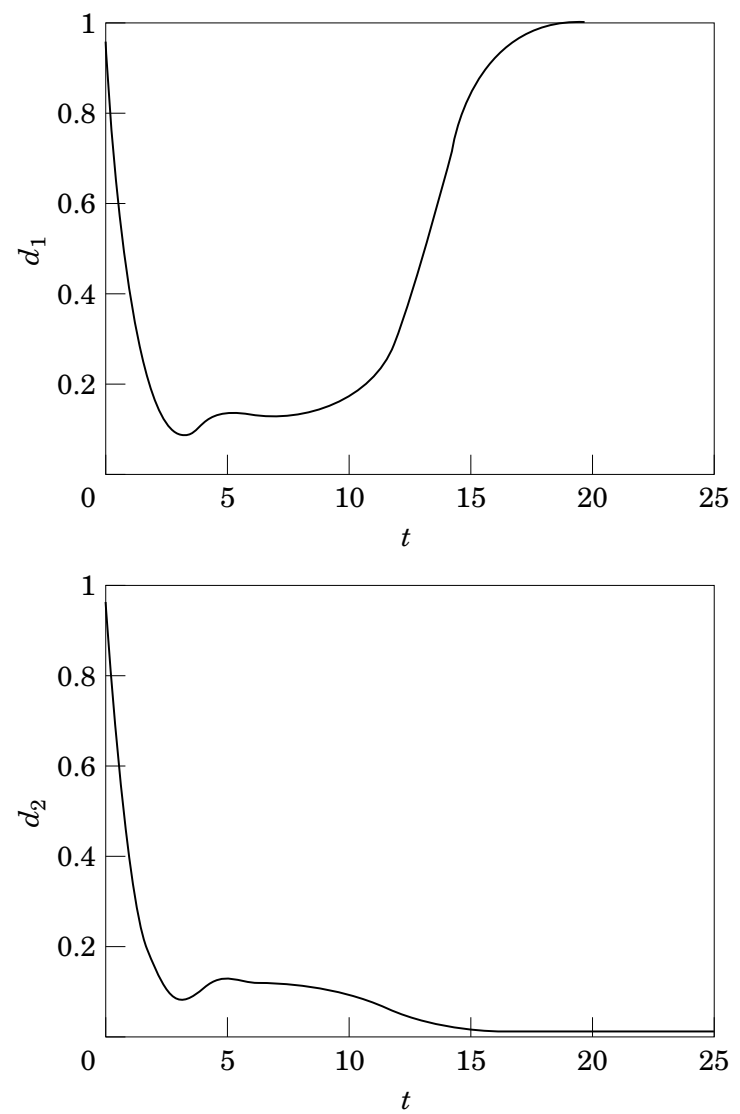

FIG. 3. A typical solution of (2) with two cells and zero boundary conditions. Parameters used are given in (5), and three steady states exist. Initial conditions are: $n_{1}(0)=1, d_{1}(0)=1, n_{2}(0)=0.99, d_{2}(0)=0.99$. 

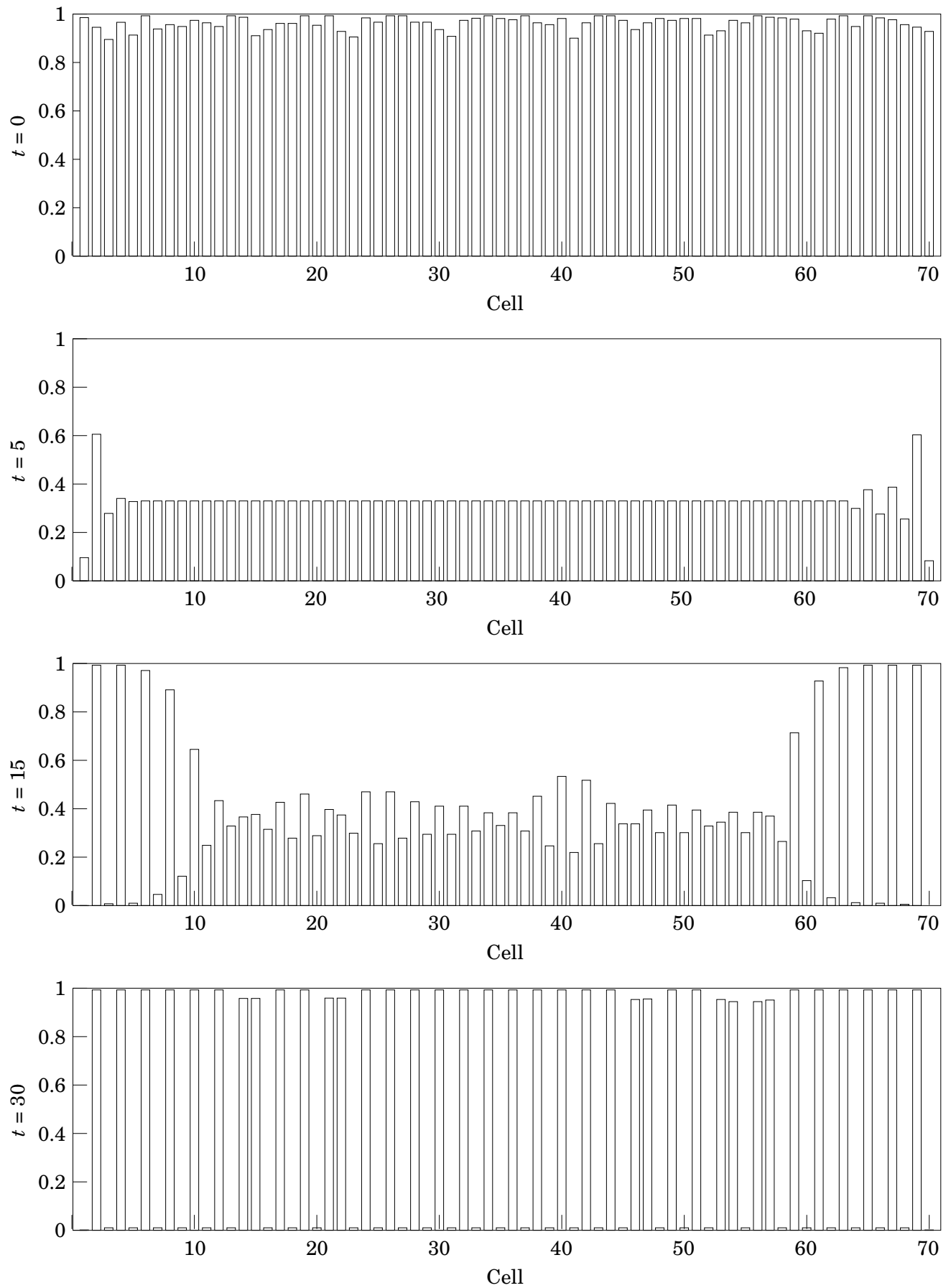

FIG. 4. Graphs showing the level of Notch activity in a line of 70 cells at successive times; $t=0,5,15,30$. (By $t=30$, the levels of Notch and Delta activity have almost reached equilibrium.) Parameters used are as in (5). Initial conditions for $n_{j}$ are shown in the top graph $(t=0)$, and $d_{j}(0)=1$ for $1 \leqslant j \leqslant 70$.

certain values of the model parameters and initial conditions, the pattern forms simultaneously across the whole domain. The model therefore exhibits behaviour characteristic of both sequential and simultaneous pattern formation (see Oster, 1988). There appears to be a characteristic "default" pattern of alternating cell fates (in one-dimension), or of the type shown in Fig. 5(a) (in two-dimensions), which forms quickly if it is compatible with the boundary conditions; defects in the pattern form (more slowly) when the default pattern is not compatible with the boundary conditions. 
The values of the parameters $a, b, k$ and $h$ determine the existence, precise values and stability of the steady states. When eqns (2) have only one steady state, the default pattern of cell fates is one in which all cells uniformly adopt an intermediate state of mutual inhibition. When the equations allow several alternative steady states, the choice of final pattern from among these possibilities is influenced by $v$ and by the initial conditions, even though these do not affect the existence, location or stability of the steady states (see Appendix A). Increasing the values of $k, h$ and/or $v$ reduces the time taken for the final pattern to emerge.

\section{ANALYSIS OF PERTURBATIONS ABOUT} HOMOGENEOUS STATES

The numerical simulations described in the previous section were performed using a particular choice of functional forms for $f$ and $g$. We have performed simulations with a range of biologicallyplausible combinations of model parameters, boundary values and (roughly homogeneous) initial data;

(a)

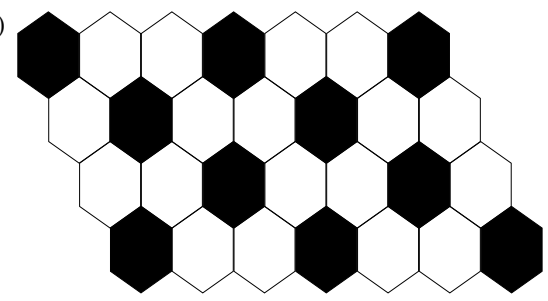

(b)

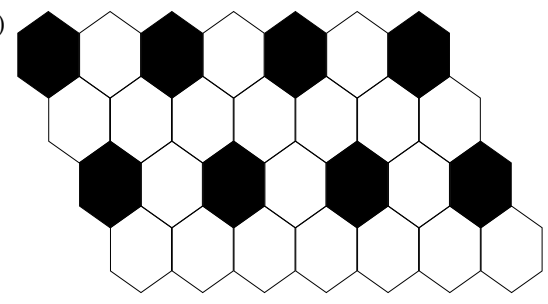

(c)

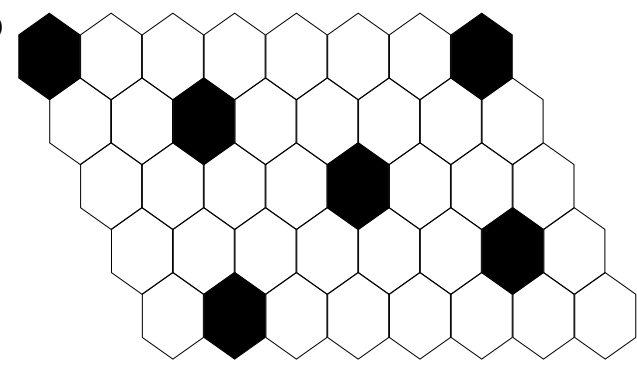

FIG. 5. Examples of periodic steady-state patterns of cells adopting the primary fate (black: low Notch activity) and secondary fate (white; high Notch activity), on a two-dimensional lattice. (a)

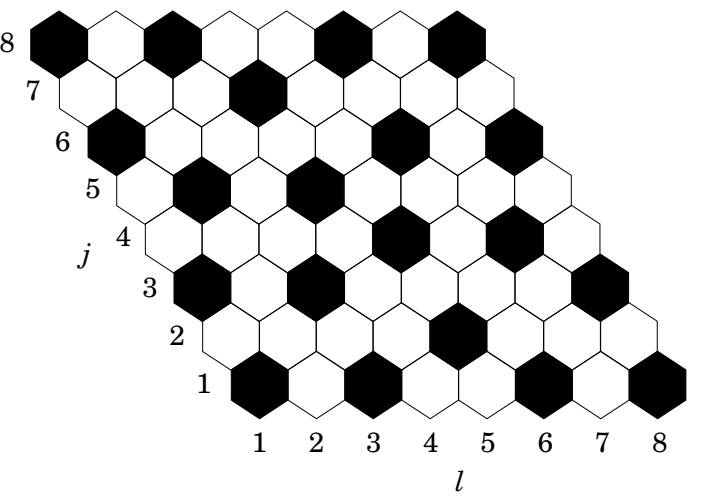

(b)

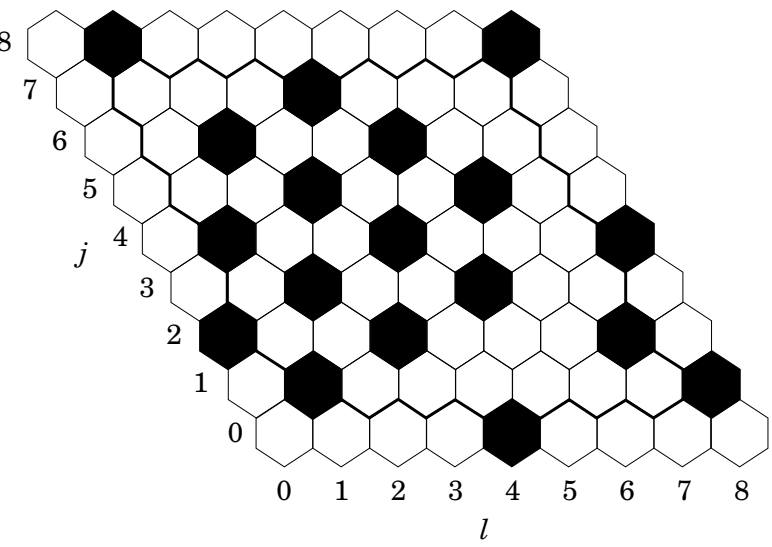

FIG. 6. Diagram showing the steady-state pattern of primary and secondary fates in an (a) $8 \times 8$ and (b) $7 \times 7$ array of hexagonal cells. Black cells will adopt the primary fate, white cells the secondary fate. Parameters used are as in (5). Boundary conditions: (a) $d_{l j}=0$ for $l, j=0$ or 9 ; (b) periodic. Initial conditions: $n_{l j}(0)$ and $d_{l j}(0)$ have arbitrary values between 0.95 and 1.0.

the patterns of cell fate that are obtained are qualitatively the same as the examples shown here. This strongly suggests that the types of pattern that emerge from the system (2) are insensitive to the precise values of the model parameters, provided that heterogeneous steady states exist.

To investigate this proposition further, we have performed a linear analysis of perturbations about the homogeneous steady state to deduce the pattern forming capabilities of an infinite system of discrete cells described by eqns (2). The formalism we use is an extension of that used by Turing (1952) to analyse the linear stability of the homogeneous steady state in a coupled system of discrete cells. However, it is important to note that while the mathematical techniques used are similar, the actual patterning mechanism we are considering is different from the diffusion-driven instability investigated by Turing. This discrete approach is well-suited to analysing systems in which values of variables change abruptly from one cell to the next. The behaviour of the system 
in the neighbourhood of the homogeneous steady state is particularly significant, given our findings that the levels of Notch and Delta activity in an initially roughly homogeneous population of cells often tend to the homogeneous steady state levels before diverging to their final (heterogeneous) equilibrium levels (see Figs. 3, 4, A2 and Appendix A).

We thus consider an array of cells which are all initially near the homogeneous steady state. To investigate the patterns that emerge from perturbations about this steady state, we linearise the system (2) about this state, and study the growth of inhomogeneities in the levels of Notch and Delta activity starting from the homogeneous values. On the assumption that these early spatial distributions of Notch and Delta activity determine the final pattern attained, this analysis enables the patterns that result from initial conditions near the homogeneous steady state to be determined. The details of this analysis for one- and two-dimensional arrays of cells are contained in Appendix B.

The results confirm and extend the results of the numerical simulations. For a one-dimensional array of cells, the analysis predicts that the default pattern of cell fates will be the period 2 pattern in which alternate cells adopt opposite fates. For a large two-dimensional array of hexagonal cells, the predicted pattern has three different cell states, although two of these can be identical (see Fig B1). Thus, the predicted patterns are in agreement with those obtained from the numerical simulations of the system. It is important to note, however, that specific forms for the functions $f$ and $g$ are not assumed in the analysis, and that it thus constitutes a generalisation of our numerical results. Our model is thus robust, in the sense that the final pattern of cell fates that emerges does not depend on the specific forms of $f$ and $g$ (and therefore on specific biochemical parameters), provided that the feedback between cells is strong enough.

\section{Discussion}

We have investigated the pattern-forming potential of a dynamical model of Delta-Notch intercellular signalling, based on a feedback loop that acts to amplify differences in the levels of activity of Notch and Delta between adjacent cells. Numerical simulations show that this system is capable of generating coherent patterns of Notch and Delta activity from random initial data. For a wide range of initial conditions and parameter values, a pattern characteristic of the action of short-range lateral inhibition emerges; cells in which levels of Notch activation are low are surrounded by cells with a high level of Notch activation. Cells with low levels of Notch activity (and hence high levels of Delta activity) are not found next to each other. This is in concordance with experimental findings on the spatial distribution of cells adopting primary and secondary fates. By an extension to infinite one- and two-dimensional domains of the linear analysis used by Turing (1952), we have analysed the emergence of patterns for generic forms of the interaction between Notch and Delta. This analysis complements and confirms the numerical results.

The feedback loop on which the model presented here is based has two major components. Firstly, adjacent cells interact through Delta-Notch signalling in such a way that the rate of increase of Notch activity in a cell is an increasing function of the amount of Delta signalling received from all adjacent cells. Secondly, the rate of increase of Delta activity in a cell is a decreasing function of the level of Notch activity in that cell.

The formulation of this model for lateral inhibition in terms of specific molecules, Notch and Delta, is motivated by a range of genetic and molecular data. These have established that Delta and Notch are essential parts of a mechanism mediating lateral inhibition (Lehmann et al., 1983); that both are transmembrane proteins (Wharton et al., 1985; Kidd et al., 1986; Vässin et al., 1987; Kopczynski et al., 1988); that Notch functions as a receptor in this cell-cell interaction (Heitzler \& Simpson, 1991, 1993; Artavanis-Tsakonas et al., 1995); and that Delta is a ligand for Notch such that Delta-Notch binding leads to activation of the Notch signalling pathway (Fehon et al., 1990; Heitzler \& Simpson, 1991; Rebay et al., 1993; Lieber et al., 1993; Struhl et al., 1993). It has been convincingly shown, moreover, that activation of the Notch signalling pathway in a cell leads to repression of Delta signalling from that cell (Heitzler \& Simpson, 1991, 1993; Heitzler et al., 1996), providing the feedback that plays an essential part in our model. The details of the mechanism of this repression remain uncertain, although there is evidence that transcriptional regulation is involved, operating via Suppressor of Hairless (Fortini \& Artavanis-Tsakonas, 1994; Schweisguth, 1995), products of the Enhancer of split complex (Jennings et al., 1995; Heitzler et al., 1996) and products of the achaete-scute complex (Heitzler et al., 1996; Hinz et al., 1994; Kunisch et al., 1994). There is increasing evidence that binding of Delta to Notch causes Notch itself to be proteolytically cleaved, releasing an intracellular fragment that moves into the nucleus 
and initiates the transcriptional cascade (Jarriault et al., 1995; Lieber et al., 1993; Struhl et al., 1993).

Given that there are still uncertainties about the control of Delta activity, we have avoided committing ourselves to a specific detailed set of assumptions. Instead, we have considered here a general mathematical form for the relationship between levels of Notch and Delta activity in a cell. This is justified, since our analytical results show that the qualitative predictions of the model are insensitive to the exact form of the relationship, provided that the feedback is sufficiently strong. Indeed, since we make only minimal assumptions about the details of the feedback interaction between cells, our model is also applicable to nearest-neighbour feedback interactions between cells that are mediated by factors other than Notch and Delta, provided that the feedback mechanism satisfies the assumptions we have made.

\section{Conclusions}

The insensitivity of our model to the details of the biochemistry allows us to draw general conclusions about the pattern-forming potential of a Delta-Notch feedback loop:

(1) The feedback loop is capable of generating spatial patterns from random stochastic fluctuations in a population of initially equivalent cells. Provided that the feedback is strong enough, homogeneous states are unstable to small fluctuations. No "prepattern" is required. Moreover, the pattern of cell fates generated by the model is robust.

(2) The feedback loop generates a "competition" between members of a population of initially equivalent cells. The feedback loop amplifies small stochastic differences between cells, creating a situation in which each cell is either chiefly a deliverer of inhibition (the primary fate) or chiefly a recipient of inhibition (the secondary fate). Because the eventual levels of Notch and Delta activities in a cell are determined through competitive signalling between neighbouring cells, situations in which adjacent cells both adopt the primary fate are avoided. In contrast, there is no clear reason why such situations should not occur if cells are singled out for the primary fate by some prior, cell-autonomous mechanism before lateral inhibition begins to operate (as has sometimes been suggested-see, for example, Artavanis-Tsakonas et al., 1995). However, adjacent cells can both adopt the secondary fate, since in this case the signalling between the cells is very weak (see, for example, Fig. 4).

(3) The form of feedback loop considered here can account only for fine-grained patterns of cell specialisation. Our model postulates that interactions occur only between nearest neighbour cells. Such short-range inhibition does indeed appear to be operating in a number of cell fate decisions [for example, in the Drosophila endoderm (Tepass \& Hartenstein, 1995) and peripheral nervous system proneural clusters (Heitzler \& Simpson, 1991, 1993)] and our model accounts well for these examples. However, the model is unable to account for longer-range patterns whose characteristic scale at the time of cell determination is of the order of many cell diameters.

Of course, if cell divisions ensue after the fine-grained pattern has been set up, the final pattern in the full-grown organism can show a larger spacing. The pattern of microchaetae (small bristles) in the Drosophila epidermis probably arises in this way, as a result of Delta-Notch-mediated lateral inhibition followed by growth (Simpson, 1990). But where the scale of the pattern at the time of cell determination is of the order of many cell diameters, our arguments imply that other mechanisms instead of, or in addition to, the type of Delta-Notch signalling considered here must be operating. For example, in many cases, such as that of the macrochaetae (large bristles) on a fly, these more widely-spaced patterns represent a prior inhomogeneous pattern of expression of proneural genes such as achaete and scute (Skeath \& Carroll, 1991).

Other models for the generation of lateral inhibition-type patterns have been proposed previously. These fall into two general classes, based on reaction-diffusion and "neural" mechanisms (Murray, 1993; Oster, 1988). We feel that a particular attraction of the model presented here is that it is based on well-defined and plausible assumptions about the nature of the molecular factors involved. An added advantage of our approach is that it is very well suited to further extension, for example to include the effects of other cell-autonomous and non-autonomous factors on the Delta-Notch feedback loop. Factors believed to interact with the Delta-Notch-mediated lateral inhibition machinery include secreted molecules such as the Wingless protein (Couso \& Martínez Arias, 1994; González-Gaitán \& Jäckle, 1995; Axelrod et al., 1996) and asymmetrically localised intracellular determinants such as Numb and Prospero (Doe \& Spana, 1995). As the number 
of such factors increases, the complexity of the genetic interactions between them makes it imperative to consider quantitative models of their actions. The model presented here provides a versatile framework on which to base further, more extensive models.

JRC and NAMM contributed equally to this work and gratefully acknowledge the support of the Wellcome Trust (grant refs. 041290/Z/94 and 041306/Z/94 respectively). JHL is indebted to the Imperial Cancer Research Fund for support. PKM would like to thank the Department of Mathematics, Williams College, Massachusetts for their support and hospitality.

\section{REFERENCES}

Artavanis-Tsakonas, S., Matsuno, K. \& Fortini, M. E. (1995). Notch signaling. Science 268, 225-268.

Austin, C. P., Feldman, D. E., Ida JR., J. A. \& Cepko, C. L. (1995). Vertebrate retinal ganglion cells are selected from competent progenitors by the action of Notch. Development 121, 3637-3650.

Axelrod, J. D., Matsuno, K., Artavanis-Tsakonas, S. \& Perrimon, N. (1996). Interaction between wingless and Notch signalling pathways mediated by dishevelled. Science 271, $1826-1832$.

Campos-Ortega, J. A. (1993). Early neurogenesis in Drosophila melanogaster. In: The Development of Drosophila melanogaster (Bate, M. \& Martínez-Arias, A., eds) Vol. 2, pp. 1091-1129. New York: Cold Spring Harbor Laboratory Press.

Chitnis, A. B. (1995). The role of Notch in lateral inhibition and cell fate specification. Mol. Cell. Neurosci. 6, 311-321.

Chitnis A., Henrique, D., Lewis, J., Ish-Horowicz, D. \& Kintner, C. (1995). Primary neurogenesis in Xenopus embryos regulated by a homologue of the Drosophila neurogenic gene Delta. Nature 375, 761-766.

Couso, J. P. \& Martínez Arias, A. (1994). Notch is required for wingless signaling in the epidermis of Drosophila. Cell 79, 259-272.

Doe, C. Q. \& Spana, E. P. (1995). A collection of cortical crescents: asymmetric protein localization in CNS precursor cells. Neuron 15, 991-995.

Fehon, R. G., Kooh, P. J., Rebay, I., Regan, C. L., Xu, T., Muskavitch, M. A. T. \& Artavanis-Tsakonas, S. (1990). Molecular interactions between the protein products of the neurogenic loci Notch and Delta, two EGF-homologous genes in Drosophila. Cell 61, 523-534.

Fortini, M. E. \& Artavanis-Tsakonas, S. (1994). The Suppressor of Hairless protein participates in Notch receptor signalling. Cell 79, 273-282.

GonZÁleZ-Gaitan, M. \& JÄCKLE, H. (1995). Invagination centers within the Drosophila stomatogastric nervous system anlage are positioned by Notch-mediated signaling which is spatially controlled through wingless. Development 121, 2313-2325.

Goriely, A., Dumont, N., Dambly-Chaudière, C. \& Ghysen, A. (1991). The determination of sense organs in Drosophila: effect of neurogenic mutations in the embryo. Development 113, $1395-1404$

Greenwald, I. \& Rubin, G. M. (1992). Making a difference: the role of cell-cell interactions in establishing separate identities for equivalent cells. Cell 68, 271-281.

Hartenstein, V. \& Posakony, J. W. (1990). A dual function of the Notch gene in Drosophila sensillium development. Dev. Biol. 142, 13-30.

Heitzler, P., Bourouis, M., Ruel, L., Carteret, C. \& Simpson, P. (1996). Genes of the Enhancer of split and achaete-scute complexes are required for a regulatory loop between Notch and Delta during lateral signalling in Drosophila. Development 122, 161-171.
Heitzler, P. \& Simpson, P. (1991). The choice of cell fate in the epidermis of Drosophila. Cell 64, 1083-1092.

Heitzler, P. \& Simpson, P. (1993). Altered epidermal growth factor-like sequences provide evidence for a role of Notch as a receptor in cell fate decisions. Development 117, 1113-1123.

Hinz, U., Giebel, B. \& Campos-Ortega, J. A. (1994). The basic helix-loop-helix domain of Drosophila lethal of scute protein is sufficient for proneural function and activates neurogenic genes. Cell 76, 77-87.

Jarriault, S., Brou, C., Logeat, F., Schroeter, E. H., Kopan, R. \& IsRael, A. (1995). Signalling downstream of activated mammalian Notch. Nature 377, 355-358.

Jennings, B., de Celis, J., Delidakis, C., Preiss, A. \& Bray, S. (1995). Role of Notch and achaete-scute complex in the expression of Enhancer of split bHLH proteins. Development 121, 3745-3752.

Kidd, S., Kelley, M. R. \& Young, M. W. (1986). Sequence of the Notch locus of Drosophila melanogaster: relationship of the encoded protein to mammalian clotting and growth factors. $\mathrm{Mol}$. Cell. Biol. 6, 3094-3108.

Kopczynski, C. C., Alton, A. K., Fechtel, K., Кooh, P. J. \& Muskavitch, M. A. T. (1988). Delta, a Drosophila neurogenic gene, is transcriptionally complex and encodes a protein related to blood coagulation factors and epidermal growth factor of vertebrates. Genes Dev. 2, 1723-1735.

Kunisch, M., Haenlin, M. \& Campos-Ortega, J. A. (1994). Lateral inhibition mediated by the Drosophila neurogenic gene Delta is enhanced by proneural proteins. Proc. Natl. Acad. Sci. U.S.A. 91, 10139-10143.

Lehmann, R., Jimenez, F., Dietrich, U. \& Campos-Ortega, J. A. (1983). On the phenotype and development of mutants of early neurogenesis in Drosophila melanogaster. Roux's Arch. Dev. Biol. 192, 62-74.

LEWIS, J. (1996). Neurogenic genes and vertebrate neurogenesis. Curr. Opin. Nerobiol. 6, 3-10.

Lieber, T., Kidd, S., Alcamo, E., Corbin, V. \& Young, M. W. (1993). Antineurogenic phenotypes by truncated Notch proteins indicate a role in signal transduction and may point to a novel function for Notch in nuclei. Genes Dev. 7, 1949-1965.

Murray, J. D. (1993). Mathematical Biology. 2nd Edn. Berlin: Springer-Verlag.

Muskavitch, M. A. T. (1994). Delta-Notch signaling and Drosophila cell fate choice. Dev. Biol. 166, 415-430.

Oster, G. (1988). Lateral inhibition models of developmental processes. Math. Biosci. 90, 265-286.

Rebay, I., Fehon, R. G. \& Artavanis-Tsakonas, S. (1993). Specific truncations of Drosophila Notch define dominant activated and dominant negative forms of the receptor. Cell 74, 319-329.

Schweisguth, F. (1995). Suppressor of Hairless is required for signal reception during lateral inhibition in the Drosophila pupal notum. Development 121, 1875-1884.

Simpson, P. (1990). Lateral inhibition and the development of the sensory bristles of the adult peripheral nervous system of Drosophila. Development 109, 509-519.

Skeath, J. B. \& CARRoll, S. B. (1992). Regulation of proneural gene expression and cell fate during neuroblast segregation in the Drosophila embryo. Development 114, 939-946.

Sternberg, P. W. (1993). Falling off the knife edge. Curr. Biol. 3, $763-765$.

Struhl, G., Fitzgerald, K. \& Greenwald, I. (1993). Intrinsic activity of the lin-12 and Notch intracellular domains in vivo. Cell 74, 331-345.

TePass, U. \& Hartenstein V. (1995). Neurogenic and proneural genes control cell fate specification in the Drosophila endoderm. Development 121, 393-405.

Turing, A. M. (1952). The chemical basis of morphogenesis. Phil. Trans. R. Soc. 237, 37-72.

VÄssin, H., Bremer, K. A., Knust, E. \& CAmpos-Ortega, J. (1987). The neurogenic gene Delta of Drosophila melanogaster is expressed in neurogenic territories and encodes a putative transmembrane protein with EGF-like repeats. $E M B O J .6$, $3433-3440$ 
Wharton, K. A., Johansen K. M., Xu, T. \& Artavanis-Tsakonas, S. (1985). Nucleotide sequence from the neurogenic locus Notch implies a gene product that shares homology with proteins containing EGF-like repeats. Cell 43, 567-581.

Wilkinson, H. A., Fitzgerald, K. \& Greenwald, I. (1994). Reciprocal changes in expression of the receptor lin-12 and its ligand lag-2 prior to commitment in a $C$. elegans cell fate decision. Cell 79, 1187-1198.

\section{APPENDIX A}

\section{Analysis of the two-cell system}

We consider the non-dimensional form (2) of the basic equations

$$
\begin{gathered}
\dot{n}_{P}=f\left(\bar{d}_{P}\right)-n_{P}, \\
\dot{d}_{P}=v\left\{g\left(n_{P}\right)-d_{P}\right\} .
\end{gathered}
$$

For a system consisting of two cells with periodic boundary conditions, these become

$$
\begin{aligned}
& \dot{n}_{1}=f\left(d_{2}\right)-n_{1}, \dot{d}_{1}=v\left\{g\left(n_{1}\right)-d_{1}\right\}, \\
& \dot{n}_{2}=f\left(d_{1}\right)-n_{2}, \dot{d}_{2}=v\left\{g\left(n_{2}\right)-d_{2}\right\},
\end{aligned}
$$

where the subscripts correspond to cells 1 and 2 .

\section{STEADY STATES AND LINEAR STABILITY}

Steady states of the system (6) are given by:

$$
\left(n_{1}^{*}, d_{1}^{*}, n_{2}^{*}, d_{2}^{*}\right)=\left(n_{1}^{*}, g\left(n_{1}^{*}\right), n_{2}^{*}, g\left(n_{2}^{*}\right)\right),
$$

where $n_{1}^{*}$ and $n_{2}^{*}$ are fixed points of $f g f g$ with $n_{1}^{*}=f g\left(n_{2}^{*}\right)$ and $n_{2}^{*}=f g\left(n_{1}^{*}\right)$.

Since $f, g:[0, \infty) \rightarrow[0, \infty)$ are continuous and $f g$ is monotonic decreasing, there exists $x_{0} \in[0, f g(0)]$ such that $x_{0}=f g\left(x_{0}\right)$, and $x_{0}$ is the unique fixed point of $f g$. Hence there is exactly one homogeneous steady state:

$$
\left(n_{1}^{*}, d_{1}^{*}, n_{2}^{*}, d_{2}^{*}\right)=\left(x_{0}, g\left(x_{0}\right), x_{0}, g\left(x_{0}\right)\right) .
$$

There may also be one or more pairs of opposite heterogeneous steady states. Suppose $\left(x_{1}, x_{2}\right)$ is a period 2 solution of the map $z_{i+1}=f g\left(z_{i}\right)$. Then $x_{1}=f g\left(x_{2}\right), x_{2}=f g\left(x_{1}\right)$, and $x_{1} \neq x_{2}$, so this gives a pair of heterogeneous steady states:

$\left(n_{1}^{*}, d_{1}^{*}, n_{2}^{*}, d_{2}^{*}\right)=\left\{\begin{array}{l}\left(x_{1}, g\left(x_{1}\right), x_{2}, g\left(x_{2}\right)\right), \text { or } \\ \left(x_{2}, g\left(x_{2}\right), x_{1}, g\left(x_{1}\right)\right) .\end{array}\right.$

In particular, if $f$ and $g$ are continuously differentiable on $[0, \infty)$, then when $(f g)^{\prime}\left(x_{0}\right)<-1$, where ' denotes differentiation, there must be at least one period 2 solution $\left(x_{1}, x_{2}\right)$ of the map $z_{i+1}=f g\left(z_{i}\right)$. If $x_{1}<x_{0}$ then $x_{2}>x_{0}$. This can be seen intuitively from Fig. A1. Provided $f$ and $g$ are smooth functions, standard linear stability analysis shows that a steady state $\left(n_{1}^{*}, d_{1}^{*}, n_{2}^{*}, d_{2}^{*}\right)$ is linearly stable if
$\mathscr{M}:=(f g f g)^{\prime}\left(n_{1}^{*}\right)<1$, but unstable if $\mathscr{M}>1$. Even when the steady state is linearly unstable, any temporal oscillations decay.

For the homogeneous steady state $\left(x_{0}, g\left(x_{0}\right)\right.$, $\left.x_{0}, g\left(x_{0}\right)\right)$,

$$
\mathscr{M}=(f g)^{\prime}\left(f g\left(x_{0}\right)\right) \cdot(f g)^{\prime}\left(x_{0}\right)=\left[(f g)^{\prime}\left(x_{0}\right)\right]^{2}
$$

and so the condition for linear instability reduces to $(f g)^{\prime}\left(x_{0}\right)<-1$. Thus, whenever the homogeneous steady state is linearly unstable [i.e. $(f g f g)^{\prime}\left(x_{0}\right)>1$ ], there exists a pair of heterogeneous steady states of the form given in (A.1). The converse is not necessarily true.

As a model for lateral inhibition, we are most interested in the case where the homogeneous steady state is unstable and there exists a pair of stable heterogeneous steady states. If $x_{1}<x_{2}$, then the steady state $\left(x_{1}, g\left(x_{1}\right), x_{2}, g\left(x_{2}\right)\right)$ corresponds to cell 1 adopting the primary fate, (with a low level of Notch activity), and cell 2 adopting the secondary fate. If the feedback between Delta and Notch is weak, $(f g f g)^{\prime}(x)$ will be less than one for all $x$, and so the homogeneous steady state will be the only steady state. If the feedback is sufficiently strong [for example, if $\left.(f g)^{\prime}\left(x_{0}\right)<-1\right]$, then there will exist heterogeneous steady states, and initially equivalent neighbouring cells may diverge to different fates. Thus, the final pattern of cell fates that emerges from random initial data depends on the strength of the Delta-Notch feedback loop.

\section{PHASE PLANE ANALYSIS FOR V $\ll 1$}

If levels of Notch activity respond to changes in Delta activity much more rapidly than levels of Delta activity respond to changes in Notch activity, then $v$ will be very small compared to one. In this case, phase plane analysis can be used to study the long term behaviour of the system (A.1).

On a slow timescale $T=v t, \quad \mathrm{~d} n_{j} / \mathrm{d} t=v \mathrm{~d} n_{j} /$ $\mathrm{d} T:=v n_{j}^{\prime}=O(v), \quad$ (for $j=1,2$ ), after fast initial transients. Hence we make the quasi-steady-state assumption that

$$
f\left(d_{2}\right)-n_{1}=0, \text { and } f\left(d_{1}\right)-n_{2}=0,
$$

when $T=O(1)$. Then the system (A.1) reduces to:

$$
d_{1}^{\prime}=g f\left(d_{2}\right)-d_{1}, d_{2}^{\prime}=g f\left(d_{1}\right)-d_{2},
$$

for $T=O(1)$, to lowest order in $v$.

Since $g f$ is a monotonic decreasing function on $[0, \infty)$, there is always a homogeneous steady state $\left(d_{1}^{*}, d_{2}^{*}\right)=\left(g\left(x_{0}\right), g\left(x_{0}\right)\right)$, and there may also be pairs of heterogeneous steady states. As in the previous 
section, a steady state $\left(d_{1}^{*}, d_{2}^{*}\right)$ is linearly stable if $\mathscr{M}<1$, where $\mathscr{M}=(f g f g)^{\prime}\left(n_{1}^{*}\right)$, and $n_{1}^{*}=f\left(d_{2}^{*}\right)$.

The $\left(d_{1}, d_{2}\right)$ phase plane is symmetric about the line $d_{1}=d_{2}$, which forms a separatrix, separating trajectories in the region $d_{1}<d_{2}$ from those in the region $d_{1}>d_{2}$. When the homogeneous steady state $\left(g\left(x_{0}\right), g\left(x_{0}\right)\right)$ is a saddle point, this line is its stable invariant manifold. Typical phase planes are sketched in Fig. A2, where the forms in (4) are used for $f$ and $g$.

Figure A2 shows that when $v \ll 1$, it is the initial values of $d_{1}$ and $d_{2}$ that determine which of the two opposite heterogeneous steady states will be attained eventually. An analogous analysis to that presented here can be performed for $v \gg 1$, in which case it is found that it is the initial values of $n_{1}$ and $n_{2}$ that determine the final state attained (data not shown). These results suggest that there is a separatrix in the full $\left(n_{1}, d_{1}, n_{2}, d_{2}\right)$ phase space [i.e. that relating to the solutions of the full system (A1)], the position of which depends on $v$, separating those trajectories which converge to $\left(x_{1}, g\left(x_{1}\right), x_{2}, g\left(x_{2}\right)\right)$ from those which converge to $\left(x_{2}, g\left(x_{2}\right), x_{1}, g\left(x_{1}\right)\right)$. Consequently, the final state to which solutions of the full system settle would be determined by the parameter $v$, for some sets of initial conditions. This is supported by numerical results for the full system.

\section{ANALYSIS OF PERTURBATIONS ABOUT A GENERAL HOMO- GENEOUS STATE}

To investigate the behaviour of small perturbations about a general homogeneous state, consider the two-cell system governed by (A1) and change to the variables:

$$
\begin{aligned}
\mathscr{N} & =\left(n_{1}+n_{2}\right) / 2, \quad \mathscr{D}=\left(d_{1}+d_{2}\right) / 2, \\
x & =\left(n_{1}-n_{2}\right) / 2, \quad y=\left(d_{1}-d_{2}\right) / 2,
\end{aligned}
$$

(a)

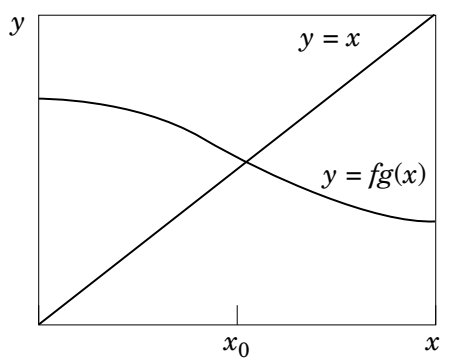

(b)

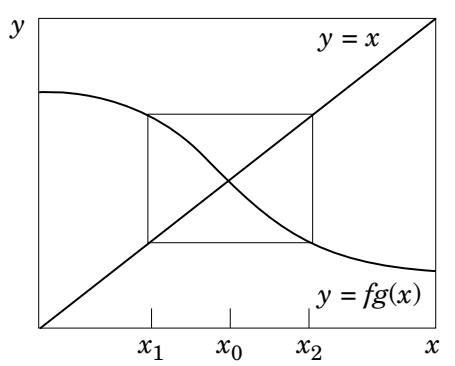

(c)

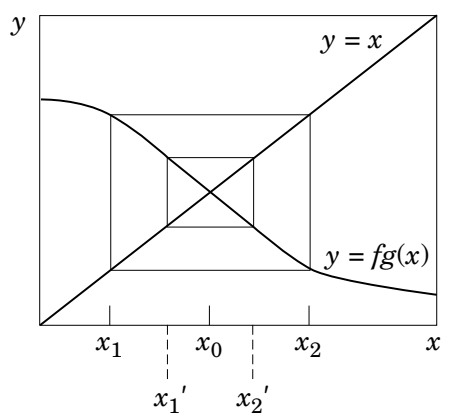

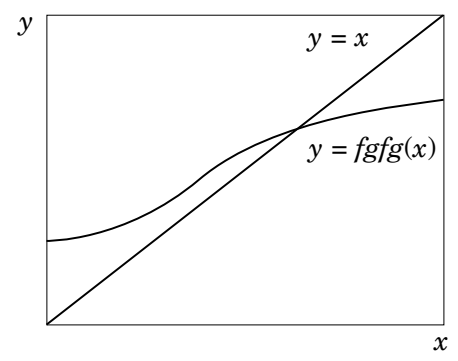
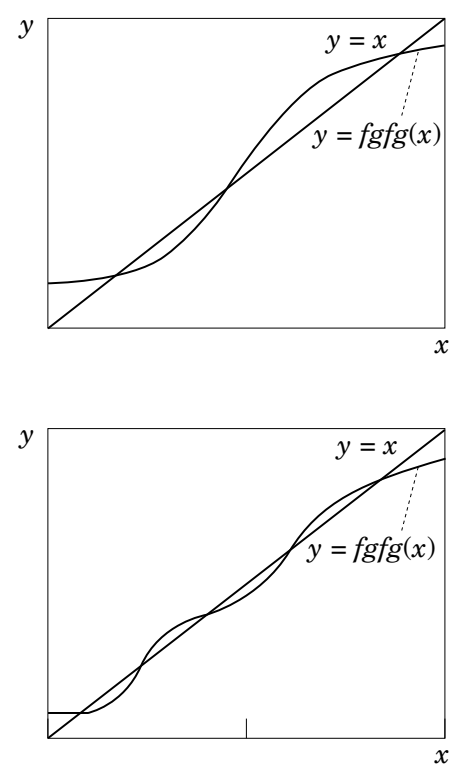

FIG. A1. Examples showing the existence of different numbers of steady states. In (a), the only steady state is the homogeneous one, with $n_{1}^{*}=n_{2}^{*}=x_{0}$. In (b), there are three steady states; the homogeneous one, and two opposite heterogeneous ones with $n_{1}^{*}=x_{1}, n_{2}^{*}=x_{2}$ or $n_{1}^{*}=x_{2}, n_{2}^{*}=x_{1}$. In (c), there are five steady states; the homogeneous one and two pairs of heterogeneous solutions; $\left\{n_{1}^{*}, n_{2}^{*}\right\}=\left\{x_{1}\right.$, $\left.x_{2}\right\}$ or $\left\{x_{1}^{\prime}, x_{2}^{\prime}\right\}$. See text for details. 

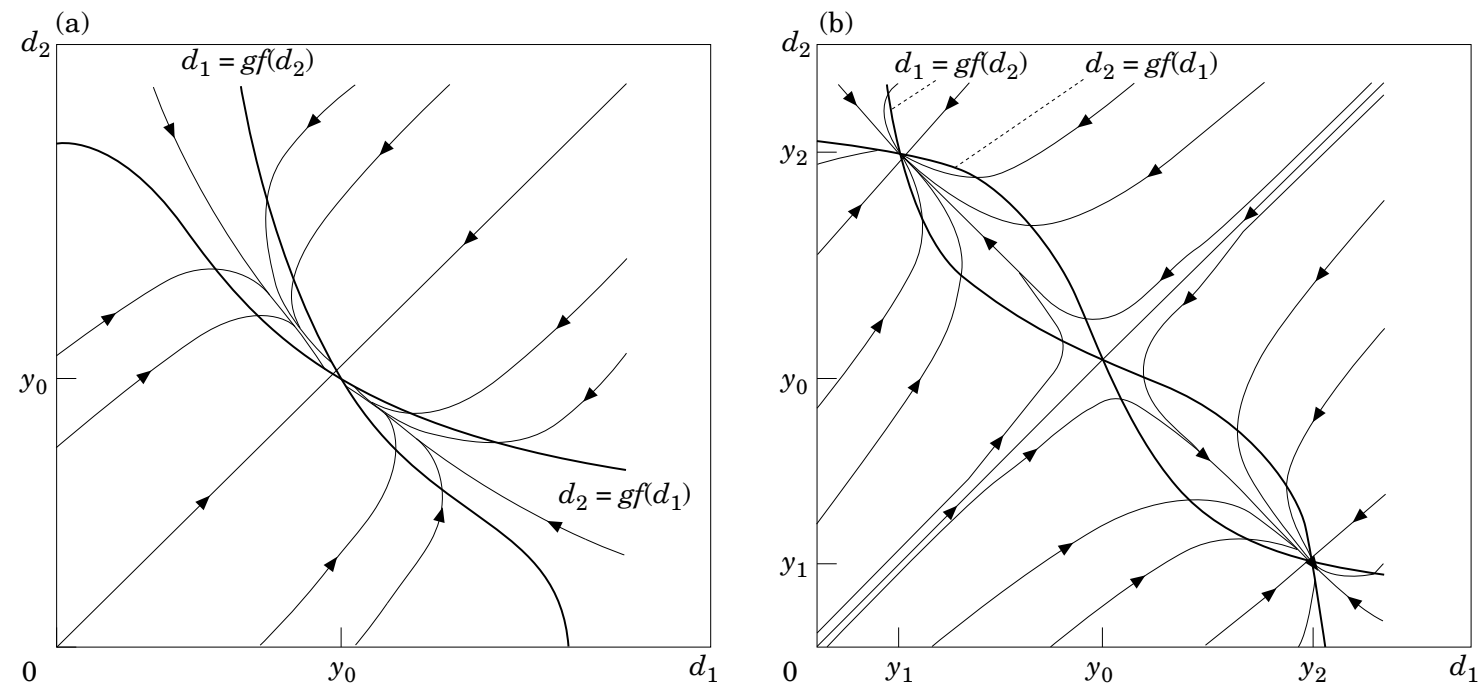

FIG. A2. Phase planes for the two cell system when $v \ll 1 . \dot{d}_{1}=0$ on the null cline $d_{1}=g f\left(d_{2}\right)$, and $\dot{d}_{2}=0$ on $d_{2}=g f\left(d_{1}\right)$. In (a) there is only one steady state, which is linearly stable since $(f g)^{\prime}\left(x_{0}\right)>-1$. In (b) there are three steady states; the homogeneous one is an unstable saddle point, while the two heterogeneous steady states are stable nodes. $y_{i}=g\left(x_{i}\right)$ for $i=0,1,2$.

where $|x|$ and $|y|$ are small. Then, to first order in $x$ and $y$,

$$
\begin{gathered}
\dot{N}=f(\mathscr{D})-\mathscr{N}, \dot{\mathscr{D}}=v(g(\mathscr{N})-\mathscr{D}), \\
\dot{x}=-f^{\prime}(\mathscr{D}) y-x, \dot{y}=v\left(g^{\prime}(\mathscr{N}) x-y\right) .
\end{gathered}
$$

The equations for $\mathscr{N}$ and $\mathscr{D}$ decouple from those for $x$ and $y$, and can be solved (in principle) to give $\mathscr{N}$ and $\mathscr{D}$ as functions of time. Let

$$
f^{\prime}(\mathscr{D})=r(t), v g^{\prime}(\mathscr{N})=s(t) .
$$

From the initial postulates of our model, $f^{\prime}(\mathscr{D}) \geqslant 0$, $\forall \mathscr{D}$ and $g^{\prime}(\mathscr{N}) \leqslant 0, \forall \mathscr{N}$.

Setting

$$
\mathbf{z}=\left[\begin{array}{l}
x \\
y
\end{array}\right]
$$

the linearised equations for $x$ and $y$ can be written as:

$$
\frac{\mathrm{d}}{\mathrm{d} t}(\mathbf{z})=H(t) \mathbf{z},
$$

where

$$
H(t)=\left[\begin{array}{cc}
-1 & -r(t) \\
s(t) & -v
\end{array}\right]
$$

We can thus draw "instantaneous phase portraits", showing the rates of change of $x$ and $y$ at each instant $t$. When $f^{\prime}(\mathscr{D})$ and $g^{\prime}(\mathscr{N})$ vary slowly relative to $x$ and $y$, these give an indication of the behaviour of $x$ and $y$ over a short timescale. The phase portraits fall into two classes, depending on the sign of $f^{\prime}(\mathscr{D}) g^{\prime}(\mathscr{N})+1$. Representative examples of these two classes are shown in Fig. A3, in which the arrows indicate the instantaneous rates of change of $x$ and $y$.

From Fig. A3 it can be seen that a necessary condition for both $|x|$ and $|y|$ to be growing in magnitude at some instant is that

$$
f^{\prime}(\mathscr{D}) g^{\prime}(\mathscr{N})<-1 .
$$

These instantaneous phase portraits can be used to piece together a qualitative picture of the overall behaviour of a system that is initially close to homogeneity. Consider a two-cell system in which levels of Delta and Notch activity are initially roughly equal in the two cells, and are both high or both low. Then if $f$ and $g$ are of the form given in (4), $f^{\prime}(\mathscr{D}) g^{\prime}(\mathscr{N})+1$ will initially be positive, and so the instantaneous phase portrait will be of the form shown in Fig. A3(a). Thus, any initial inhomogeneity between the cells will tend to decrease, and the overall behaviour of the system will be dominated by the behaviour of $\mathscr{N}$ and $\mathscr{D}$. These will tend towards their steady state values, $\mathscr{N}^{*}$ and $\mathscr{D}^{*}$, given by $\mathscr{N}^{*}=x_{0}$ and $\mathscr{D}^{*}=g\left(x_{0}\right)$. As a consequence, the levels of Delta and Notch activity in the cells will tend towards their values at the homogeneous steady state.

However, as $\mathscr{N}$ and $\mathscr{D}$ change in this way, the value of $f^{\prime}(\mathscr{D}) g^{\prime}(\mathscr{N})+1$ steadily decreases. In terms of the instantaneous phase portraits, the null clines (i.e. the curves $\dot{x}=0$ and $\dot{y}=0$ ) move closer together until, when $f^{\prime}(\mathscr{D}) g^{\prime}(\mathscr{N})+1=0$, they eventually cross, and the instantaneous phase portrait becomes of the form shown in Fig. A3(b). Now inhomogeneities between the cells can grow, and eventually become the dominant factor in determining the behaviour of the 
system. Behaviour of this kind is exhibited in the simulation shown in Fig. 3.

It is important to note that since $f^{\prime}(\mathscr{D})$ and $g^{\prime}(\mathscr{N})$ change with time, the $(x, y)$ phase plane also changes with time. In particular, the relative positions of the null clines $\dot{x}=0$ and $\dot{y}=0$ are governed by the sign of $f^{\prime}(\mathscr{D}) g^{\prime}(\mathscr{N})+1$. Thus, in general, it is not possible to determine the behaviour of perturbations over long time scales from the instantaneous phase portraits. However, we can determine conditions on $\mathscr{N}$ and $\mathscr{D}$ under which the magnitude, $|\mathbf{z}|$, of all small perturbations from homogeneity will decay. Now,

$$
\begin{aligned}
& \frac{\mathrm{d}}{\mathrm{d} t}\left(|\mathbf{z}|^{2}\right)=\frac{\mathrm{d}}{\mathrm{d} t}\left(\mathbf{z}^{T} \mathbf{z}\right) \\
= & z^{\mathrm{T}}\left(H^{T}+H\right) \mathbf{z}=\mathbf{z}^{T} A \mathbf{z},
\end{aligned}
$$

where

$$
A=H^{T}+H=\left[\begin{array}{cc}
-2 & s-r \\
s-r & -2 v
\end{array}\right]
$$

is a real symmetric matrix. Thus $A$ can be diagonalised by a linear transformation of the form $\mathbf{z}=P \mathbf{u}$, where $P$ is an orthogonal matrix of eigenvectors of $A$. Therefore, if $A$ has eigenvalues $\lambda_{+}$ and $\lambda_{-}$, we can write

$$
\begin{aligned}
\frac{\mathrm{d}}{\mathrm{d} t}\left(|\mathbf{z}|^{2}\right) & =\mathbf{u}^{T}\left[\begin{array}{cc}
\lambda_{+} & 0 \\
0 & \lambda_{-}
\end{array}\right] \mathbf{u} \\
& =\lambda_{+} u_{1}^{2}+\lambda_{-} u_{2}^{2} .
\end{aligned}
$$
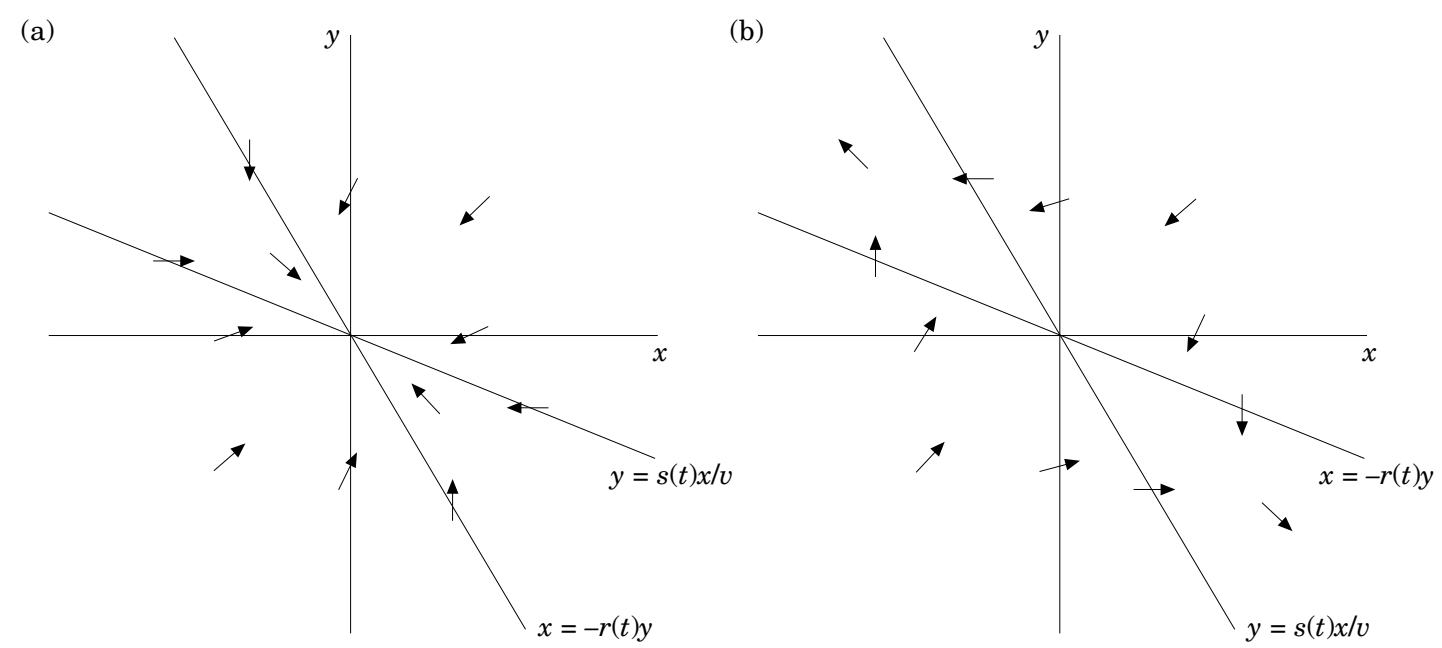

FIG. A3. "Instantaneous phase portraits", showing the behaviour of perturbations $x$ and $y$ at a given instant in time. In (a), $r(t) s(t)>-v$, while in (b) $r(t) s(t)<-v$, [where $r(t)=f^{\prime}(D)$ and $s(t)=v g^{\prime}(N)$ ]. Note that $|x|$ and $|y|$ can both be increasing only when $r(t) s(t)<-v$. Arrows indicate the instantaneous rates of change of $x$ and $y$. See text for details. 
A combination of the techniques used in this section and those developed in Appendix B can be used to analyse a system consisting of an infinite line of cells. As for the two-cell case, there exist perturbations about homogeneity which are growing in magnitude in each cell (at a given instant), when $f^{\prime}(\mathscr{D})-v g^{\prime}(\mathscr{N})>2 \sqrt{v}$. However, the magnitudes of the perturbations in each cell have an upper bound which is decreasing when (A.3) holds (results not shown). This analysis predicts that for a long line of cells, perturbations from homogeneity may transiently decrease in magnitude even when the homogeneous steady state is unstable. An example of this type of behaviour is shown in Fig. 4.

\section{APPENDIX B}

\section{Analysis of Perturbations of the Homogeneous Steady State}

We consider the general non-dimensional form of the basic equations given in (2):

$$
\begin{aligned}
\dot{n} & =f\left(\bar{d}_{P}\right)-n_{P}, \\
\frac{1}{v} \dot{d}_{P} & =g\left(n_{P}\right)-d_{P},
\end{aligned}
$$

for each cell $P$. We assume only that $f, g$ : $[0, \infty) \rightarrow[0, \infty)$ are continuously differentiable, with $f$ monotonic increasing and $g$ monotonic decreasing; it is not necessary to consider specific forms for $f$ and $g$. Under these conditions there exists exactly one homogeneous steady state, with $n_{P}=x_{0}, d_{P}=g\left(x_{0}\right)$ for all $P$.

Consider, now, an array of cells which are all initially near the homogeneous steady state. We wish to determine the patterns that emerge from perturbations about this steady state. We assume that

$$
\left|n_{P}-x_{0}\right|,\left|d_{P}-g\left(x_{0}\right)\right| \ll 1, \forall P,
$$

and linearise the system (2) about the homogeneous steady state to determine the patterns of Notch and Delta activity that develop as heterogeneity appears. On the assumption that these early spatial distributions of Notch and Delta activity determine the final pattern attained, this analysis enables us to determine all the patterns that can be formed by the system (2), from initial conditions near the homogeneous steady state.

For the forms of $f$ and $g$ in (4), this analysis allows us to consider the patterns emerging from any initial conditions in which the cells are virtually equivalent, since the analysis in Appendix A and the results of our numerical simulations (see Section 3) show that initially similar levels of Notch and Delta activity first tend to the homogeneous steady state levels. If the homogeneous steady state is unstable, the levels of Notch and Delta activity in neighbouring cells then diverge to their final heterogeneous steady-state values.

\section{ONE-DIMENSIONAL ARRAY}

We consider an infinite domain, and look for a pattern with period $N(>1)$. For each $j=1,2, \ldots, N$, we make a change of variables which will lead to the decoupling of the system of $2 N$ equations into pairs of equations:

$$
\begin{gathered}
n_{j}-x_{0}=\sum_{s=1}^{N} \xi_{s} e^{2 \pi i s j / N}, \\
d_{j}-g\left(x_{0}\right)=\sum_{s=1}^{N} \eta_{s} e^{2 \pi i s j / N},
\end{gathered}
$$

where, for $1 \leqslant r \leqslant N$,

$$
\begin{gathered}
\xi_{r}=\frac{1}{N} \sum_{j=1}^{N}\left(n_{j}-x_{0}\right) e^{-2 \pi i r j / N}, \\
\eta_{r}=\frac{1}{N} \sum_{j=1}^{N}\left(d_{j}-g\left(x_{0}\right)\right) e^{-2 \pi i r j / N} .
\end{gathered}
$$

The system (2) can be linearised about the homogeneous steady state to give:

$$
\begin{aligned}
& \frac{\mathrm{d}}{\mathrm{d} t}\left(n_{j}-x_{0}\right) \\
& =\frac{a}{2}\left[\left(d_{j-1}-g\left(x_{0}\right)\right)+\left(d_{j+1}-g\left(x_{0}\right)\right)\right]-\left(n_{j}-x_{0}\right), \\
& \frac{\mathrm{d}}{\mathrm{d} t}\left(d_{j}-g\left(x_{0}\right)\right)=v b\left(n_{j}-x_{0}\right)-v\left(d_{j}-g\left(x_{0}\right)\right),
\end{aligned}
$$

where $a:=f^{\prime}\left(g\left(x_{0}\right)\right)$ and $b:=g^{\prime}\left(x_{0}\right)$. We assume that $a>0>b$. Using these equations, the expressions in (B.1) yield

$$
\begin{aligned}
\frac{\mathrm{d} \xi_{r}}{\mathrm{~d} t} & =a \cos (2 \pi r / N) \eta_{r}-\xi_{r}, \\
\frac{\mathrm{d} \eta_{r}}{\mathrm{~d} t} & =v b \xi_{r}-v \eta_{r}
\end{aligned}
$$

This gives us a pair of linear equations with constant coefficients for each $r=1,2, \ldots, N$. The equations for different values of $r$ are uncoupled. Standard 
analysis shows that the general solution to (B.2) is of the form

$$
\begin{aligned}
{\left[\begin{array}{c}
n_{j}-x_{0} \\
d_{j}-g\left(x_{0}\right)
\end{array}\right] } & =\sum_{N=1}^{\infty} \sum_{s=1}^{N} e^{2 \pi i s j / N} \\
\times & \left(\left[\begin{array}{c}
A_{s, N}^{+} \\
B_{s, N}^{+}
\end{array}\right] \exp \left[\lambda_{s, N}^{+} t\right]+\left[\begin{array}{c}
A_{s, N}^{-} \\
B_{s, N}^{-}
\end{array}\right] \exp \left[\lambda_{s, N}^{-} t\right]\right),
\end{aligned}
$$

where the $\lambda_{s, N}^{ \pm}$and $\left[\begin{array}{c}A_{s, N}^{ \pm} \\ B_{s, N}^{ \pm}\end{array}\right]$

are the eigenvalues and eigenvectors of the matrix

$$
\left[\begin{array}{cc}
-1 & a \cos (2 \pi s / N) \\
v b & -v
\end{array}\right]
$$

There are several points to note from this general solution.

(1) If $\lambda_{s, N}^{ \pm} \notin \mathbb{R}$ then $\operatorname{Re}\left(\lambda_{s, N}^{ \pm}\right)=-\delta(1+v) / 2<0$, so any temporal oscillations decay. Hence we do not expect wave-like or temporally oscillating solutions to dominate in the full nonlinear system.

(2) The homogeneous steady state $n_{j}=x_{0}, d_{j}=g\left(x_{0}\right)$ is linearly stable if and only if $\operatorname{Re}\left(\lambda_{s, N}^{ \pm}\right)<0 \forall s$, $N$; i.e. if and only if $1>|a b|$, or

$$
\left|(f g)^{\prime}\left(x_{0}\right)\right|=\left|f^{\prime}\left(g\left(x_{0}\right)\right) \cdot g^{\prime}\left(x_{0}\right)\right|<1 .
$$

(3) The eigenvalues with the largest real part are

$$
\lambda_{N / 2, N}^{+}=\frac{1}{2}\left\{-(1+v)+\sqrt{(1-v)^{2}-4 v a b}\right\},
$$

where $N$ is even (the eigenvalues corresponding to odd $N$ have smaller real parts). These are real since $a b<0$.

Hence the dominant pattern for large $t$ is a superposition of modes with even periodicity where $s / N=1 / 2$. These modes have parallel eigenvectors, so we find that

$$
\begin{array}{r}
{\left[\begin{array}{c}
n_{j}-x_{0} \\
d_{j}-g\left(x_{0}\right)
\end{array}\right] \sim C(-1)^{j}\left[\begin{array}{l}
A \\
B
\end{array}\right]} \\
\exp \left[\frac{t}{2}\left\{-(1+v)+\sqrt{(1-v)^{2}-4 v a b}\right\}\right],
\end{array}
$$

as $t \rightarrow+\infty$, where

$$
\left[\begin{array}{l}
A \\
B
\end{array}\right]:=\left[\begin{array}{c}
\frac{1}{2}\left\{-(1-v)+\sqrt{(1-v)^{2}-4 v a b}\right\} \\
v b
\end{array}\right],
$$

and $C$ is an arbitrary constant.

Our conclusions tie in well with the analysis of the two-cell case and with the numerical simulations. When $(f g)^{\prime}\left(x_{0}\right)<-1$, the homogeneous steady state is unstable, and a spatially oscillating solution with period 2 emerges, in which each cell has either a high level of Notch activity and a low level of Delta activity or vice versa. Adjacent cells have opposite fates. Small perturbations grown exponentially, (without temporal oscillations), on a time scale of order

$$
2 /\left\{-(1+v)+\sqrt{(1-v)^{2}-4 v a b}\right\},
$$

which is positive for $a b<-1$. This is in agreement with the results of our numerical simulations.

\section{TWO-DIMENSIONAL ARRAY OF HEXAGONAL CELLS}

We linearise (2) and look for spatially-periodic solutions of the form

$$
\begin{gathered}
n_{l j}-x_{0}=\sum_{s=1}^{N} \sum_{r=1}^{M} \xi_{r s} e^{2 \pi i s j / N+2 \pi i r l / M}, \\
d_{l j}-g\left(x_{0}\right)=\sum_{s=1}^{N} \sum_{r=1}^{M} \eta_{r s} e^{2 \pi i s j / N+2 \pi i r / / M},
\end{gathered}
$$

where, for $1 \leqslant q \leqslant M$ and $1 \leqslant p \leqslant N$,

$$
\begin{gathered}
\xi_{q p}=\frac{1}{M N} \sum_{l=1}^{M} \sum_{j=1}^{N}\left(n_{l j}-x_{0}\right) e^{-2 \pi i(p j / N+q l / M)}, \\
\eta_{q p}=\frac{1}{M N} \sum_{l=1}^{M} \sum_{j=1}^{N}\left(d_{l j}-g\left(x_{0}\right)\right) e^{-2 \pi i(p j / N+q l / M)} .
\end{gathered}
$$

Then,

$$
\begin{aligned}
\frac{\mathrm{d} \xi_{q p}}{\mathrm{~d} t}=\frac{a}{3}(\cos (2 \pi(q / M+p / N)) \\
+\cos (2 \pi q / M)+\cos (2 \pi p / N)) \eta_{q p}-\xi_{q p}, \\
\frac{\mathrm{d} \eta_{q p}}{\mathrm{~d} t}=v b \xi_{q p}-v \eta_{q p} .
\end{aligned}
$$

These equations can be solved in the same way as in the one-dimensional array. Again, any temporal oscillations decay. However, there are differences between the two cases: 
(1) The homogeneous steady state is linearly stable on a two-dimensional array of hexagonal cells if and only if

$$
\left|(f g)^{\prime}\left(x_{0}\right)\right|=|a b|<2 .
$$

Thus, the homogeneous steady state is stable for larger values of $|a b|$ than in a one-dimensional array of cells.

(2) The eigenvalues with largest real part are those for which $M$ and $N$ are multiples of three, and $q / M=p / N=1 / 3$ or $q / M=p / N=2 / 3$; i.e.

$$
\begin{aligned}
\lambda_{M / 3, N / 3, M, N}^{+} & =\lambda_{2 M / 3,2 N / 3, M, N}^{+} \\
& =\frac{1}{2}\left\{-(1+v)+\sqrt{(1-v)^{2}-2 v a b}\right\} .
\end{aligned}
$$

For large $t$, the solution of the linearised problem is asymptotically like:

$$
\begin{aligned}
& {\left[\begin{array}{c}
\eta_{l j}-x_{0} \\
d_{l j}-g\left(x_{0}\right)
\end{array}\right] \sim\left\{C e^{2 \pi i(j+t) / 3}+D e^{4 \pi i(j+t) / 3}\right\} } \\
\times & {\left[\begin{array}{c}
\tilde{A} \\
B
\end{array}\right] \exp \left[\frac{t}{2}\left\{-(1+v)+\sqrt{(1-v)^{2}-2 v a b}\right\},\right.}
\end{aligned}
$$

where

$$
\left[\begin{array}{l}
\tilde{A} \\
B
\end{array}\right]:=\left[\begin{array}{c}
\frac{1}{2}\left\{-(1-v)+\sqrt{(1-v)^{2}-2 v a b}\right\} \\
v b
\end{array}\right],
$$

and $C, D$ are arbitrary constants.

This solution grows or decays exponentially on a timescale of order

$$
2 /\left|\left\{-(1+v)+\sqrt{(1-v)^{2}-2 v a b}\right\}\right|,
$$

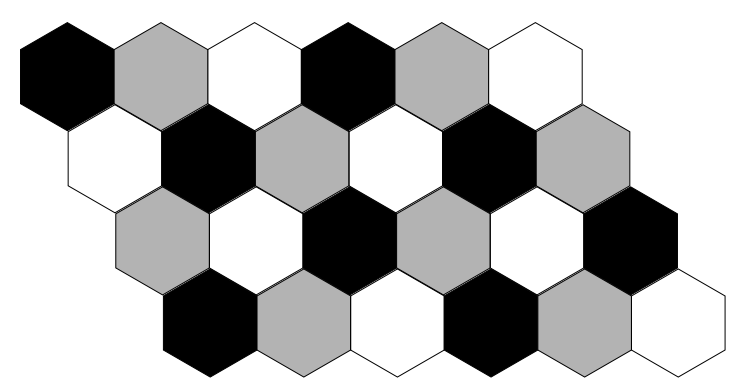

FIG. B1. Generic pattern of Notch activity on an infinite two-dimensional array of hexagonal cells, as predicted by linear analysis. There may be one, two or three different cell types, arranged as shown. Cells with the same degree of shading are of the same type, but two or three of the types may be identical. The pattern has periodicity 3 in $j+l$ (see Fig. 2 for cell labelling scheme).

which is slower than in a one-dimensional array.

The main difference between (B.5) and the analogous solutions in the one-dimensional array is in the spatial variation. The dominant pattern has periodicity 3 in $j+l$, (rather than 2), and having a linear combination of two independent spatial oscillations allows a greater variety of patterns. The generic pattern is illustrated in Fig. B1. There are three types of cell (according to whether $j+l=0,1$ or 2 mod 3), although two of these types may be identical (if $C=D$, for example).

This analysis predicts that for a large two-dimensional array of cells the generic pattern has periodicity 3 in $j+l$. Thus pattern (a) in Fig. 5 (with a ratio of secondary to primary fate cells of 2) would be possible, but not patterns (b) and (c). Other patterns may be possible with three different cell types, (as illustrated in Fig. B1), but the linear analysis here does not tell us which of these patterns emerges in the full, nonlinear system. 